\title{
A Graph Realization Approach to Network Identification
}

\author{
Marzieh Nabi-Abdolyousefi, Maryam Fazel, and Mehran Mesbahi
}

\begin{abstract}
In this paper, we examine the problem of identifying the interaction geometry among a known number of agents, adopting a consensus-type algorithm for their coordination. The proposed identification process is facilitated by introducing "ports" for steering a subset of network vertices via an appropriately defined interface and observing the network's response at another set of vertices. Using the fact that system identification provides a realization of the original network, we utilize transformations to identify a graph topology that is compatible with the set of input-output data. An example demonstrates the application of the proposed method.
\end{abstract}

Keywords: Inverse problems; coordination algorithms; network identification

\section{INTRODUCTION}

Physical sciences are often concerned with inferring models and physical parameters from data. Given a model for a physical phenomena, computing the data values is often referred to as the forward problem. On the other hand, in inverse problems, the objective is the construction, validation, invalidation, or reconstruction of the model from a set of measurements associated with the system. Inverse problems arise in fields such as astronomy, geophysics, medical imaging, remote sensing, ocean acoustic tomography, and nondestructive testing [1], [2].

Closer to the present work are the inverse problems associated with electrical networks [3], and the celebrated "Can one hear the shape of a drum?" which aims to characterize a manifold via its spectra [4], or more recently, "Can one hear the shape of a graph?" [5]. In fact, in this paper, we address the inverse problem related to consensus-type coordination algorithms. Consensus-type algorithms have recently been employed for analysis and synthesis of a host of distributed protocols and control strategies in multi-agent systems, including, flocking, formation control, rendezvous, and distributed estimation [6].

One of the key aspects of consensus-type protocols is the strong dependency between the interaction and informationexchange geometry among the multiple agents, on one hand, and the dynamic properties that these systems exhibit, on the other. Motivated by this dependency, in our work, we consider the scenario where the interaction network is inside a "black box," and that only certain "boundary" nodes in the network can be influenced and subsequently observed. The "input" boundary nodes are then used to stimulate the network, whose response is subsequently observed at the "output" boundary nodes. Using this setup, in our complementary work [7], we have presented a node knockout

The research was supported by NSF grant CMMI-0856737 and AFOSR grant FA9550-12-1-0203-DEF. Emails: \{mnabi, mfazel, mesbahi\}@uw.edu. procedure that aims to find the generating function of the graph Laplacian from the observed input-output data. Our focus in [8], in the meantime, was to reduce the search space for the identification of the network topology by blending ideas from system identification, integer partitioning, and degree-based graph reconstruction. The implicit contribution of our analysis is its ramifications for exact identification, up to an automorphism, from boundary nodes for networks that have an embedded consensus-type algorithm for their operation, including formation flying, distributed estimation, and mobile robotics.

There are a number of research works addressing the problem of identifying an unknown underlying graph topology from a data set [9], [10], [11], [12], [13], [14], [15], [16]. More recently, Materassi et al. in [17] and [18] have provided an exact reconstruction procedure for tree-like network topologies. Ayazoglu et al. in [19] have proposed an optimization scheme to recover the sparsest topology (in the sense of having the fewest number of links) based on an experimental noisy data. Moreover, Sanandaji et al. in [20], inspired by the emerging field of compressive sensing, have shown that suitably sparse networks can be exactly identified from small numbers of node observations. In [20], the authors have assumed that interconnected nodes are coupled by a discrete-time convolution process.

In the present work, we adopt a system identification procedure for identifying the underlying network topology. This will be pursued by assuming that the network is observable and controllable, and aim to characterize an appropriate similarity transformation that can be applied to the system realization- obtained from the system identification. Subsequently, utilizing the fact that the similarity transformation between two negative semi-definite matrices is unitary, as well as features of graph Laplacians for weighted and unweighted networks, we proceed to construct the appropriate unitary transformation that can be used on the realization of the system matrices in order to identify the graph topology. We also address some of the numerical aspects of the proposed approach.

Our notation and terminology are standard. ${ }^{1}$ We denote by $\mathcal{G}=(\mathcal{V}, \mathcal{E})$ the undirected simple graph with vertex set $\mathcal{V}$ and edge set $\mathcal{E}$, comprised of two-element subsets of $\mathcal{V}$; we use "nodes" or "agents" interchangeably with "vertices." Two vertices $u, v \in \mathcal{G}$ are called adjacent if $\{u, v\} \in \mathcal{E}$. For vertex $i, \operatorname{deg} i$ denotes the number of its adjacent vertices or neighbors. The Laplacian matrix for the graph $\mathcal{G}$ is denoted

\footnotetext{
${ }^{1}$ The main focus of this work is on undirected graphs. However, the extension of some results to the directed case will be examined in subsequent works.
} 
by $L(\mathcal{G})$ and the weighted Laplacian by $L_{w}(\mathcal{G})$. Laplacian matrices are positive semi-definite whose spectrum will be ordered as $0=\lambda_{1}(L(\mathcal{G})) \leq \lambda_{2}(L(\mathcal{G})) \leq \ldots \leq \lambda_{n}(L(\mathcal{G}))$, and equivalently, $0=\lambda_{1}\left(L_{w}(\mathcal{G})\right) \leq \lambda_{2}\left(L_{w}(\mathcal{G})\right) \leq \ldots \leq$ $\lambda_{n}\left(L_{w}(\mathcal{G})\right)$. We use $\phi_{\mathcal{G}}(s)$ to denote the characteristic polynomial of the graph Laplacian. The cardinality of the set $\mathcal{H}$ will be denoted by $|\mathcal{H}| ; M^{T}$ and $\|M\|_{F}$ denote the transpose and Frobenius norm of matrix $M$, respectively. $M_{i, j}$ denotes the $i j-t h$ entry of the matrix $M$.

\section{NETWORK IDENTIFICATION}

Consider the consensus protocol adopted by $n$-nodes, where $x_{i}$ is the state of the $i$-th node, e.g., its position, speed, heading, voltage, etc., evolves according to the sum of the differences between the $i$-th node's state and its neighbors. Next, let a group of agents $\mathcal{I} \subset \mathcal{V}$ with cardinality $|\mathcal{I}|=r_{\mathcal{I}}$, "excite" the underlying coordination protocol by injecting signals to the network, with another set of agents $\mathcal{O} \in \mathcal{V}$, of cardinality $|\mathcal{O}|=r_{\mathcal{O}}$, measuring the corresponding network response. Hence, the original consensus protocol from node $i$ 's perspective assumes the form

$$
\dot{x}_{i}(t)=\sum_{\{i, j\} \in \mathcal{E}} w_{i j}\left(x_{j}(t)-x_{i}(t)\right)+B_{i} u_{i}(t),
$$

where $w_{i j}$ is the weight on the edge $\{i, j\}$ and $B_{i}=\beta_{i}$ if $i \in \mathcal{I}$, and zero otherwise. Without loss of generality, we can always assume that $\beta_{i}=1$ and modify the control signal $u_{i}(t)$ as $\beta_{i} u_{i}(t)$ if necessary. Adding the observation ports to this "steered" consensus, and having $y_{j}(t)=x_{j}(t)$ when $j \in \mathcal{O}$, we arrive at the compact form of an input-output linear time-invariant system,

$$
\dot{x}(t)=A(\mathcal{G}) x(t)+B u(t), \quad y(t)=C x(t),
$$

where $A(\mathcal{G})=-L_{w}(\mathcal{G}) \in \mathbf{R}^{n \times n}, B \in \mathbf{R}^{n \times r_{\mathcal{I}}}$, and $C \in$ $\mathbf{R}^{r_{\mathcal{O}} \times n}$.

Even though in general sets $\mathcal{I}$ and $\mathcal{O}$ can be distinct and contain more than one element, for the convenience of our presentation, we will assume that they are identical. The extension of the presented results to the case when $\mathcal{I}$ and $\mathcal{O}$ are distinct will be discussed as a difference could potentially occur.

We now pose the inverse problem of graph-based coordination algorithms, namely, the feasibility of identifying the spectral and structural properties of the underlying network $\mathcal{G}$ via the data facilitated by the input-output ports $\mathcal{I}$ and $\mathcal{O}$. In order to implement this program, however, we need to assume that: (1) the identification procedure has knowledge of the number of agents in the network, and (2) the input/output sets $\mathcal{I}$ and $\mathcal{O}$ have been chosen such that the system described in (2) is controllable and observable. Although the first assumption is reasonable in general, the second one requires more justification which we now provide. In the trivial case when $\mathcal{I}=\mathcal{V}$ and $B$ is equal to the identity matrix, the input-output consensus (2) is controllable and by duality, observable. However, more generally, the controllability/observability of the network from a subset of its boundary nodes, is less trivial, and more to the point, not guaranteed for general graphs [6]. In the meantime, since we will need controllability and observability of the network for its identifiability, we will rely on a topical conjecture in the algebraic graph theory to the effect that for large values of $n$, the ratio of graphs with $n$ nodes that are not controllable from any single node to the total number of graphs on $n$ nodes approaches zero as $n \rightarrow \infty$ [21].

In the present paper, we take the controllability and the observability of the underlying graph from the input and output nodes as our working assumption. In the meantime it is always convenient to know when the network is uncontrollable from a given node [7], [8].

\section{A. System Identification}

We now consider a few standard system identification procedures in the context of identifying the spectra of the underlying graph Laplacian, and subsequently, gaining insights into the interconnection structure that underscores the agents' coordinated behavior.

System identification methods are implemented via sampling of the system (2) at discrete time instances, ${ }^{2}$ $\delta, 2 \delta, \ldots, k \delta, \ldots$, with $\delta>0$, assuming the form

$$
z(k+1)=A_{d} z(k)+B_{d} v(k), \quad w(k)=C_{d} z(k),
$$

where $z(k)=x(k \delta), v(k)=u(k \delta), w(k)=y(k \delta), A_{d}=$ $e^{\delta A}, B_{d}=\left(\int_{0}^{\delta} e^{A t} d t\right) B$, and $C_{d}=C{ }^{3}$ In fact, the system identification process leads to a realization of the model

$$
\widetilde{z}(k+1)=\widetilde{A}_{d} \widetilde{z}(k)+\widetilde{B}_{d} u(k), \quad \widetilde{w}(k)=\widetilde{C}_{d} \widetilde{z}(k),
$$

where $\left(\widetilde{A}_{d}, \widetilde{B}_{d}, \widetilde{C}_{d}\right)$ is the realization of $\left(A_{d}, B_{d}, C_{d}\right)$ in (3). The realized system (4), on the other hand, is equivalent to the continuous-time system

$$
\dot{\widetilde{x}}(t)=\widetilde{A} \widetilde{x}(t)+\widetilde{B} u(k), \quad y(t)=\widetilde{C} \widetilde{x}(t),
$$

with $\widetilde{A}_{d}=e^{\delta \widetilde{A}}, \widetilde{B}_{d}=\left(\int_{0}^{\delta} e^{\widetilde{A} t} d t\right) \widetilde{B}$, and $\widetilde{C}_{d}=\widetilde{C}$; in this case, $\widetilde{A}=(1 / \delta) \log _{M} \widetilde{A}_{d}$ where $\log _{M}$ denotes the matrix logarithm. Since the system (4) is a realization of the system (3), it follows that the estimated triplet $(\widetilde{A}, \widetilde{B}, \widetilde{C})$ is a realization of $(A, B, C)$ in (2). As a result, there exists a similarity transformation induced by the matrix $T$, such that

$$
\widetilde{A}=T A T^{-1}, \quad \widetilde{B}=T B, \text { and } \widetilde{C}=C T^{-1} .
$$

Recall that with the standing assumption of $C=B^{T}$ in (2), for the identified system matrices $(\widetilde{C}, \widetilde{A}, \widetilde{B})$ the product $\widetilde{C} \widetilde{A} \widetilde{B}=C A B$ leads to an $r \times r$ block partition of the matrix $L_{w}(\mathcal{G})$. Notice that if $B \neq C^{T}$, we still obtain $r^{2}$ entries of the matrix $L_{w}(\mathcal{G})$ which may not contain the diagonal entries. Considering the case where $C=B^{T}$, the product $\widetilde{C} \widetilde{A} \widetilde{B}$ provides the degree of the input/output nodes and the existing of an edge between them. Note that since input and output matrices, $B$ and $C$, are unknown, the labels of input/output

\footnotetext{
${ }^{2}$ The system identification methods work based on data sampling from the system. Since we aimed to identify the interaction geometry of the network, we originally considered a continuous system. Therefore, we need to discretize the system (2).

${ }^{3}$ The notation $e^{A}$ for a square matrix $A$ refers to its matrix exponential.
} 
nodes are unknown. The identification procedure proceeds with the predefined labeling for input/output nodes.

In summary, an identification procedure such as the above two methods, implemented on a controllable and observable steered-and-observed coordination protocol (2), leads to a system realization whose state matrix is similar to the underlying graph Laplacian and in particular sharing the same spectra and characteristic polynomial. However, a distinct and fundamental issue in our setup is that having found a matrix that is "similar" to the Laplacian of a network is far from having exact knowledge of the network structure itself [7]. This observation motivates the following question: to what extend does the knowledge of the spectra of the graph, combined with the knowledge of the input-output matrices, reduce the search space for the underlying interaction geometry? In this paper, we explore this question using techniques based on similarity transformations of symmetric and negative semidefinite matrices as well as optimization problems over such matrices.

\section{GRAPH IDENTIFICATION}

As we explained in the previous section, the system identification procedure provides the triplet $(\widetilde{A}, \widetilde{B}, \widetilde{C})$ which satisfies the set of equalities (6), where $T$ is the similarity transformation between the realization $(\widetilde{A}, \widetilde{B}, \widetilde{C})$ and the original set of matrices $(A, B, C)$ in (2). Our goal is thus to determine the unknown quadruple $(T, A, B, C)$, where the matrix $A$ satisfies the properties of a graph Laplacian. In the following, we proceed to propose an optimization-based approach in this paper for the network topology identification.

Consider the set of identities in (6) with known triplet $(\widetilde{A}, \widetilde{B}, \widetilde{C})$ and unknown quadruple $(T, A, B, C)$. For a symmetric matrix $A$, these set of equations are under-constrained with $n(n-1) / 2$ undetermined variables; however, the special structure of the matrix $A$ as a graph Laplacian can be considered in order to restrict degrees of freedom. The main contribution of this paper is finding the transformation matrix $T$ considering the Laplacian structure of $A$. The following theorem states a well-known result in matrix analysis which will be used in our approach [22].

Lemma III.1. If $W_{1}$ and $W_{2}$ are similar negative semidefinite matrices, the similarity transformation between them is a unitary matrix.

The proposed approach in this paper builds on the unitary transformation between two negative semi-definite (NSD) matrices, as well as the structural properties of the graph Laplacian and the Householder transformation described shortly. We note that the matrix $\widetilde{A}$ shares the same nonpositive eigenvalues of the Graph Laplacian $A$; however, for a matrix to be NSD, it also has to be symmetric with nonpositive eigenvalues [22]. Since $\widetilde{A}$ is the output of the system identification procedure, there is no guarantee that it will always be symmetric. Therefore, the next step is to find a symmetric matrix $\widetilde{A}_{s}$ that shares the same spectrum as $\widetilde{A}$ and has the minimum distance from $\widetilde{A}$ in some matrix norm.
Choosing the Frobenius norm to induce a metric on the space of matrices, the problem can be formulated as follows:

$$
\left\{\begin{array}{c}
\min _{\widetilde{A}_{s}}\left\|\widetilde{A}-\widetilde{A}_{s}\right\|_{F}^{2} \\
\widetilde{A}_{s}^{T}=\widetilde{A}_{s} \quad \text { and } \quad \operatorname{eig}\left(\widetilde{A}_{s}\right)=\operatorname{eig}(\widetilde{A}) .
\end{array}\right.
$$

Our next result provides an optimal analytic solution of the above optimization problem. This theorem constructs the nearest symmetric matrix $\widetilde{A}_{s}$ which shares the same spectrum as $\widetilde{A}$.

Theorem III.2. Let $\widetilde{A} \in \mathbf{R}^{n \times n}$ with non-positive set of eigenvalues. Consider the optimization problem

$$
\left\{\begin{array}{c}
\min _{X}\|\widetilde{A}-X\|_{F}^{2} \\
X^{T}=X \text { and } \operatorname{eig}(X)=\operatorname{eig}(\widetilde{A}),
\end{array}\right.
$$

where $X$ is negative semi-definite. Then,

$$
X=U \Lambda U^{T},
$$

where $\Lambda$ is a diagonal matrix with eigenvalues of $\widetilde{A}$ on the diagonal, $\Lambda_{i, i}=\lambda_{i}(\widetilde{A})$, and $U$ is the unitary matrix in the spectral decomposition of the symmetric part of $\widetilde{A}$, i.e., $S=$ $\left(\widetilde{A}+\widetilde{A}^{T}\right) / 2$. The unitary matrix $U$ appears in the spectral decomposition of $S$ as $S=U \Lambda_{S} U^{T}$, where $\Lambda_{S}$ is diagonal and $\Lambda_{S_{i, i}}=\lambda_{i}(S)$.

Proof: The proof is inspired by [23] where the nearest symmetric positive semidefinite matrix in the Frobenius norm to an arbitrary real matrix is characterized; however, the proposed nearest symmetric positive semidefinite matrix in [23] does not share the spectrum as the original matrix.

Let $X$ be the solution of the optimization problem (7). The Frobenius norm has the property that $\|G+H\|_{F_{\widetilde{A}}}^{2}=$ $\|G\|_{F}^{2}+\|H\|_{F}^{2}$, if $G=G^{T}$ and $H=-H^{T}$. Consider $\widetilde{A}=$ $S+K$, where $S=\left(\widetilde{A}+\widetilde{A}^{T}\right) / 2$ and $K=\left(\widetilde{A}-\widetilde{A}^{T}\right) / 2$ are the symmetric and skew symmetric parts of $\widetilde{A}$, respectively. Then we have $\|\widetilde{A}-X\|_{F}^{2}=\|S-X\|_{F}^{2}+\|K\|_{F}^{2}$, where $G=$ $S-X$ and $H=K$. The problem of minimizing $\|\widetilde{A}-X\|_{F}^{2}$, therefore, reduces to minimizing $\|S-X\|_{F}^{2}$.

Let $S=U \Lambda_{S} U^{T}$, where $U$ is unitary and the diagonal matrix $\Lambda_{S}$ is such that $\Lambda_{S_{i, i}}=\lambda_{i}(S)$. Moreover, let $Y=$ $U^{T} X U$. Then,

$$
\begin{aligned}
\|S-X\|_{F}^{2} & =\left\|U \Lambda_{S} U^{T}-X\right\|_{F}^{2} \\
& =\left\|U\left(\Lambda_{S}-U^{T} X U\right) U^{T}\right\|_{F}^{2}=\left\|\Lambda_{S}-Y\right\|_{F}^{2} \\
& =\sum_{i \neq j} y_{i j}^{2}+\sum_{i}\left(\lambda_{i}-y_{i i}\right)^{2} \geq \sum_{i}\left(\lambda_{i}-y_{i i}\right)^{2} .
\end{aligned}
$$

The lower bound above is attained, uniquely, for the matrix $Y=\Lambda=\operatorname{diag}\left(\lambda_{i}(\widetilde{A})\right)$, where $\lambda_{i}(\widetilde{A})$ is the $i$-th eigenvalue of $\widetilde{A}$ if these eigenvalues are rearranged such that $\mid \lambda_{1}(S)-$ $\lambda_{1}(\widetilde{A})|\leq| \lambda_{2}(S)-\lambda_{2}(\widetilde{A})|\leq \ldots \leq| \lambda_{n}(S)-\lambda_{n}(\widetilde{A}) \mid$. Therefore, for this choice of $Y, X=U \Lambda U^{T}$.

The next step is to show that $Y$, and consequently $X$, are unique. In order to prove this, it suffices to show $\| \Lambda_{S}-$ $\Lambda\left\|_{F}^{2} \leq\right\| \Lambda_{S}-W \Lambda W^{T} \|_{F}^{2}$ for an arbitrary unitary matrix 
$W$. Let us define $\Omega=W \Lambda W^{T}$. The definition of Frobenius norm now implies that

$$
\begin{aligned}
\left\|\Lambda_{S}-\Lambda\right\|_{F}^{2} & =\operatorname{trace}\left[\left(\Lambda_{S}-\Lambda\right)\left(\Lambda_{S}-\Lambda\right)^{T}\right] \\
& =\operatorname{trace}\left(\Lambda_{S}^{2}\right)-2 \operatorname{trace}\left(\Lambda_{S} \Lambda\right)+\operatorname{trace}\left(\Lambda^{2}\right) \\
\left\|\Lambda_{S}-\Omega\right\|_{F}^{2} & =\operatorname{trace}\left[\left(\Lambda_{S}-\Omega\right)\left(\Lambda_{S}-\Omega\right)^{T}\right] \\
& =\operatorname{trace}\left(\Lambda_{S}^{2}\right)-2 \operatorname{trace}\left(\Lambda_{S} \Omega\right)+\operatorname{trace}\left(\Omega^{2}\right) \\
& =\operatorname{trace}\left(\Lambda_{S}^{2}\right)-2 \operatorname{trace}\left(\Lambda_{S} \Omega\right)+\operatorname{trace}\left(\Lambda^{2}\right) .
\end{aligned}
$$

The last equality follows from $\|\Lambda\|_{F}^{2}=\|\Omega\|_{F}^{2}$ since $W$ is unitary.

In order to prove the claim that $Y$ and $X$ are unique, we need to show that $\operatorname{trace}\left(\Lambda_{S} \Lambda\right) \geq \operatorname{trace}\left(\Lambda_{S} \Omega\right)$. Note that all diagonal entries of $\Lambda, \Lambda_{S}$, and $\Omega$ are non-positive. Let us now consider trace $\left[\Lambda_{S}(\Omega-\Lambda)\right]$. Then,

$$
\begin{aligned}
& \operatorname{trace}\left[\Lambda_{S}(\Omega-\Lambda(\widetilde{A}))\right]=\sum_{i} \lambda_{i}(S)\left[\Omega_{i, i}-\lambda_{i}(\widetilde{A})\right]= \\
& \sum_{i}\left|\lambda_{i}(S)\right|\left[\left|\Omega_{i, i}\right|-\left|\lambda_{i}(\widetilde{A})\right|\right] \leq \\
& \left|\max _{i}\left(\lambda_{i}(S)\right)\right| \sum_{i}\left(\left|\Omega_{i, i}\right|-\left|\lambda_{i}(\widetilde{A})\right|\right)= \\
& \left|\max _{i}\left(\lambda_{i}(S)\right)\right|\left[\sum_{i}\left|\Omega_{i, i}\right|-\sum_{i} \mid \lambda_{i}(\widetilde{A})\right]=0,
\end{aligned}
$$

where the fact that $\Lambda(\widetilde{A})$ and $\Omega$ share the same set of eigenvalues imply the last equality. Therefore, $\operatorname{trace}\left[\Lambda_{S}(\Omega-\right.$ $\Lambda)] \leq 0$ and the claim is proved.

Theorem III.2 thus implies that $\widetilde{A}_{s}$, the nearest symmetric approximation of $\widetilde{A}$ sharing the same spectrum, defined in (7), is equal to matrix $X$. We thus proceed to find the similarity transformation between $\widetilde{A}$ and $\widetilde{A}_{s}$; however, it is non-trivial whether there is a similarity transformation between $(\widetilde{A}, \widetilde{B}, \widetilde{C})$ and $\left(\widetilde{A}_{s}, \widetilde{B}_{s}, \widetilde{C}_{s}\right)$. Therefore, first we verify whether there is a similarity transformation between $\widetilde{A}$ and $\widetilde{A}_{s}$ and then propose a procedure to find the desired transformation. The following theorem, explores the existence of a similarity transformation between $\widetilde{A}$ and $\widetilde{A}_{s}$.

Lemma III.3. The pair of matrices $\widetilde{A}$ and $\widetilde{A}_{s}$ are similar.

Proof: We note that there is a similarity transformation between $\widetilde{A}$ and $A$ as $\widetilde{A}=T A T^{-1}$ from the system identification procedure. We also know that two symmetric matrices $A$ and $\widetilde{A}_{s}$ share the same non-positive spectrum. On the other hand, Lemma III.1 implies that there is a unitary transformation $Q$ such that $A=Q^{T} \widetilde{A}_{s} Q$. Thus, there is a similarity transformation between $\widetilde{A}_{s}$ and $\widetilde{A}$ as $\widetilde{A}=G \widetilde{A}_{s} G^{-1}$ where

$$
G=T Q^{T} .
$$

Fig. 1 demonstrates these relationships.

As proved in Lemma III.3, there is a similarity transformation between $\widetilde{A}$ and $\widetilde{A}_{s}$. The purpose of the next step is to find the transformation matrix $G$ such that

$$
\min _{G}\left\|\widetilde{A} G-G \widetilde{A}_{s}\right\|_{2}
$$

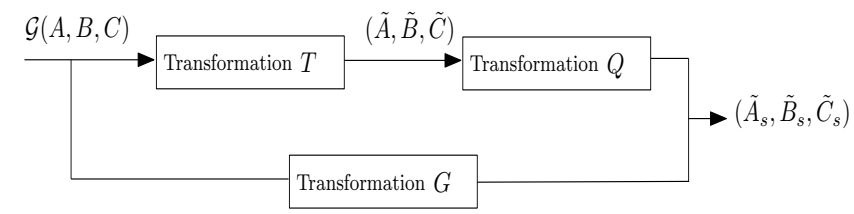

Fig. 1. Similarity transformations between $A, \widetilde{A}$, and $\widetilde{A}_{s}$

where $\widetilde{A}_{s}$ is defined in (9). Consequently, a new realization $\left(\widetilde{A}_{s}, \widetilde{B}_{s}, \widetilde{C}_{s}\right)$ is derived as

$$
\widetilde{A}_{s}=G^{-1} \widetilde{A} G, \quad \widetilde{B}_{s}=G^{-1} \widetilde{B}, \text { and } \widetilde{C}_{s}=\widetilde{C} G .
$$

As we stated earlier, in Lemma III.1, there is a unitary transformation between two NSD matrices that have the same set of eigenvalues. The next step is to blend this fact with structural features of the graph Laplacian to find the unitary transformation $Q$ between two NSD matrices $A$ and $\widetilde{A}_{s}$, i.e., $\widetilde{A}_{s} Q=Q A$. Since $A$ is the Laplacian associated with an undirected weighted graph with the property that its rows sum to zero, it follows that $\widetilde{A}_{s} Q \mathbf{1}=Q A \mathbf{1}=0$, where $\mathbf{1}$ is an $n \times 1$ vector of ones.

The equality $\widetilde{A}_{s} Q 1=0$ indicates that $Q 1$ belongs to the null space of $\widetilde{A}_{s}$. Let us denote the null space of $\widetilde{A}_{s}$ as $\widetilde{N}=$ $\operatorname{Null}\left\{\widetilde{A}_{s}\right\}$. The equality $Q 1=\widetilde{N}$ implies that the unitary matrix $Q$ rotates the vector of ones to the null space $\widetilde{N}$. The well-known Householder reflection suggests a procedure to find such a unitary matrix $Q$.

A Householder reflection, also known as Householder transformation, is a linear transformation that describes a reflection about a plane or hyperplane containing the origin. A unit vector $u$ orthogonal to the hyperplane defines the reflection hyperplane. Let

$$
Q \frac{1}{\sqrt{n}}=\frac{\widetilde{N}}{\|\widetilde{N}\|_{2}} .
$$

Then, the Householder reflection suggests that the Hermitian and unitary transformation $Q$ satisfies the following equation

$$
Q=I-2 u u^{T},
$$

where $u=v /\|v\|_{2}$ such that $v=\tilde{N} /\|\tilde{N}\|_{2}-\mathbf{1} / \sqrt{n}$.

\section{A. Numerical considerations}

Fig. 2 demonstrates a summary of the network identification procedure described above. However, there are a few related numerical analysis aspects that will be discussed in this section.

As discussed earlier, in order to calculate the matrix $Q$, it is necessary to find the null space of $\widetilde{A}_{s}$. Since $\widetilde{A}_{s}$ shares the same spectrum as $A$, it is singular and its null space, $\widetilde{N}$, is non-empty; however, numerically the null space $\widetilde{N}$ is generically empty. Therefore, we characterize the matrix $\widetilde{A}_{s}^{S}=\widetilde{A}_{s}+E$ with $E$ as the solution of the optimization problem

$$
\left\{\begin{array}{c}
\min _{E} \frac{\|E\|}{\left\|\widetilde{A}_{s}\right\|} \\
\widetilde{A}_{s}^{S^{T}}=\widetilde{A}_{s}^{S} \text { and } \widetilde{A}_{s}+E=\widetilde{A}_{s}^{S} \text { is singular, }
\end{array}\right.
$$




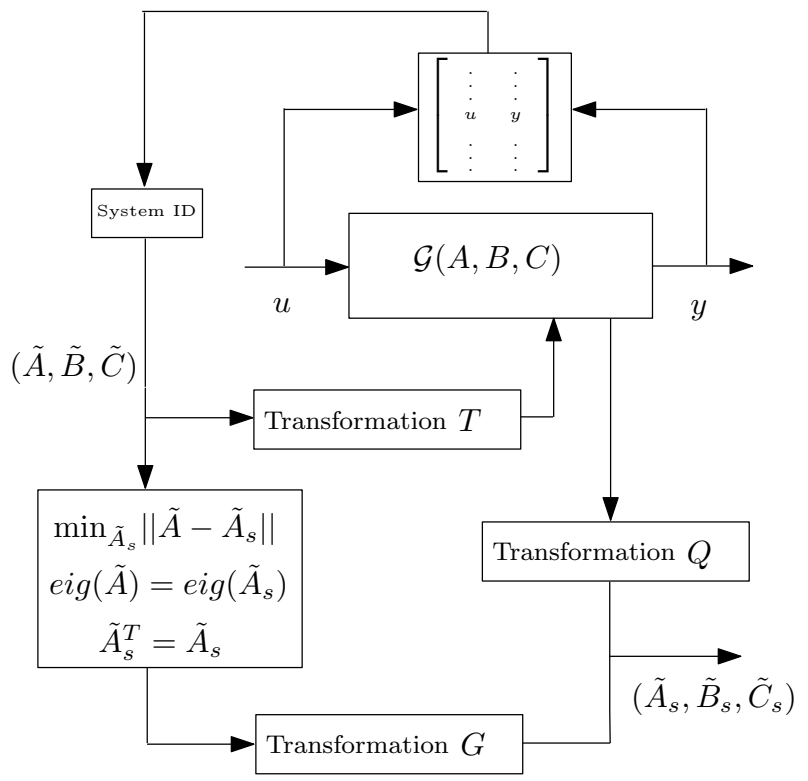

Fig. 2. Identification procedure

where $\|$.$\| is the induced matrix 2-norm. This problem is$ equivalent to finding the nearest matrix of lower rank to a given matrix [24]. The procedure for determining the nearest singular approximation of $\tilde{A}_{s}$ is inspired by [25] and [24].

Suppose that $\|$.$\| is the induced matrix norm and let z$ be a normalized vector such that $\left\|\tilde{A}_{s}^{-1} z\right\|=\left\|\tilde{A}_{s}^{-1}\right\|$ which is a valid assumption since numerical issues generically cause $\tilde{A}_{s}$ to be non-singular. Note that if the vector norm is the 2 -norm, then $z$ is the normalized eigenvector associated with the maximum eigenvalue of $\tilde{A}_{s}^{-1}$. Let

$$
w=\frac{\tilde{A}_{s}^{-1} z}{\left\|\tilde{A}_{s}^{-1}\right\|},
$$

and $v$ be a vector such that $\|v\|_{*}=1$, where $\|\cdot\|_{*}$ is the norm dual to the vector norm $\|$.$\| . Note that the dual norm$ of the 2 -norm is the 2 -norm as

$$
v^{T} w=\max _{\|u\|_{*}=1}\left|u^{T} w\right| .
$$

It then follows that $v^{T} w=\|w\|=1$ [25]; note that for the 2 -norm, it can be shown that $v=w$ [22].

Consider next the vector 2 -norm and set

$$
E=-\frac{z v^{T}}{\left\|\widetilde{A}_{s}^{-1}\right\|}=-\frac{z w^{T}}{\left\|\widetilde{A}_{s}^{-1}\right\|} .
$$

Let us define

$$
\widetilde{A}_{s}^{S}=\widetilde{A}_{s}+E=\widetilde{A}_{s}+-\frac{z w^{T}}{\left\|\widetilde{A}_{s}^{-1}\right\|} .
$$

Then,

$$
\begin{aligned}
\widetilde{A}_{s}^{S} w & =\left(\widetilde{A}_{s}+E\right) w=\widetilde{A}_{s} w-\frac{z v^{T}}{\left\|\widetilde{A}_{s}^{-1}\right\|} w \\
& =\frac{z}{\left\|\widetilde{A}_{s}^{-1}\right\|}-\frac{z}{\left\|\widetilde{A}_{s}^{-1}\right\|}=0
\end{aligned}
$$

implies that $\widetilde{A}_{s}^{S}=\widetilde{A}_{s}+E$ is singular and $w$ is in its null space.
In order to calculate $Q$ in (13), it was necessary to find the null space of $\widetilde{A}_{s}$, denoted by $\widetilde{N}$. We previously showed that $\widetilde{A}_{s}$ and $\widetilde{N}$ can be approximated by $\widetilde{A}_{s}^{S}$ and $w$, respectively. Therefore, $A=Q \widetilde{A_{s}^{S}} Q^{T}$ where $Q$ is defined in (13) and $\widetilde{A}_{s}^{S}$ in (15).

We note that $A$ can also be calculated in a different manner. The equality $G=T Q^{T}$ in (10) with known transformation $G$, as a solution of (11), implies that $T=G Q$. By finding the transformation $T$, the original matrix $A$ is thereby determined as $A=T^{-1} \widetilde{A} T$.

The approach described in this paper considers weighted consensus dynamics and provides a weighted graph that shares the same spectrum as the original weighted Laplacian with the same input/output behavior. The approach can be extended to the unweighted case, where the weights on the edges are zero or one.

In order to identify the unweighted network, we note that numerical constraints cause the calculated matrix $A$ to be a perturbed version of a Laplacian matrix even when the underlying graph is unweighted. That is, the entries of the obtained matrix are not necessarily integers. We now propose a procedure to approximate $-A$ with the closest integer matrix $L$ that satisfies the properties of a graph Laplacian.

The following mixed integer optimization problem determines the closest integer matrix $L$ that minimizes the induced matrix $2-$ norm of $A-L$ as,

$$
\min _{L}\|L-A\|_{2}
$$

subject to

$$
\begin{aligned}
& L_{i, i}>0 \\
& L_{i, j} \in\{0,-1\} \quad i \neq j \\
& L=L^{T} \\
& L \mathbf{1}=0 \\
& \sum_{i=1}^{n} L_{i, i}=-\operatorname{trace}(\widetilde{A})
\end{aligned}
$$

$L$ satisfies the constraints posed by

$$
\widetilde{C} \widetilde{A} \widetilde{B}=C A B \text { described in } \S I I-A \text {. }
$$

The proposed optimization problem can be formulated as a mixed integer least square and be solved by the CPLEX software [26]. Even though there is a subclass of mixed integer programming problems that can be solved in polynomial time, most such problems are NP-hard. This is the main pitfall associated with the application of the procedure for identification of weighted to unweighted graphs .

The implementation of the proposed methodology is discussed via an illustrative example in the next section. For this example, we study the unweighted consensus identification.

\section{AN EXAMPLE}

Our goal in this example is to gather information on the graph $\mathcal{G}$ shown in Fig. 3 running the system identification procedure. Using nodes 1,2 , and 3 as the input/output nodes in (2), we obtain $\phi_{\mathcal{G}}(s)=s^{6}+220 s^{5}+190 s^{4}+804 s^{3}+$ $1664 s^{2}+1344 s$. Since the polynomial $\phi_{G}(s)$ has just one 
zero root, the underlying graph is connected. As we discussed in [7], the characteristic polynomial $\phi_{\mathcal{G}}(s)$ reveals certain properties of the graph. For example, the graph is not a tree due to the fact that $a_{n-1} \neq 6$. Moreover, the graph has 11 edges and 224 spanning trees.

We also obtain the estimated matrices $\widetilde{A}, \widetilde{B}$, and $\widetilde{C}$ from the system identification procedure. For this example, we assume that the input and output nodes are identical. Since the diagonal of the matrix $\widetilde{C} \widetilde{A} \widetilde{B}$ is $[-3,-4,-4]^{T}, d_{1}=3$, $d_{2}=4$, and $d_{3}=3$ if we label the first three nodes as the input/output nodes. Following the proposed procedure discussed in the paper, we obtain the matrix $A_{s}$ and its nearest singular approximation $\widetilde{A}_{s}^{S}$. The transformations $Q$ is then calculated from (13). This set of data and the corresponding transformations imply that matrix $A$ and its integer Laplacian approximation $L$ are as follows

$$
\begin{aligned}
& A=\left[\begin{array}{cccc}
-3.9587 & 1.7068 & 0.4278 & 0.4031 \\
1.7068 & -4.0888 & 0.2449 & 1.1947 \\
0.4278 & 0.2449 & -3.7002 & 0.4628 \\
0.4031 & 1.1947 & 0.4628 & -3.4354 \\
0.7707 & 0.8076 & 0.7900 & 0.7497 \\
0.6504 & 0.1348 & 1.7748 & 0.6252
\end{array}\right. \\
& \begin{array}{ll}
0.7707 & 0.6504
\end{array} \\
& 0.8076 \quad 0.1348 \\
& 0.7900 \quad 1.7748 \\
& 0.7497 \quad 0.6252 \\
& \begin{array}{ll}
-3.3747 & 0.2567
\end{array} \\
& 0.2567-3.4420 \\
& L=\left[\begin{array}{cccccc}
3 & -1 & -1 & 0 & -1 & 0 \\
-1 & 4 & 0 & -1 & -1 & -1 \\
-1 & 0 & 4 & -1 & -1 & -1 \\
0 & -1 & -1 & 4 & -1 & -1 \\
-1 & -1 & -1 & -1 & 4 & 0 \\
0 & -1 & -1 & -1 & 0 & 3
\end{array}\right]
\end{aligned}
$$

Fig. 3 depicts the original graph and the identical determined graph from the proposed approach.

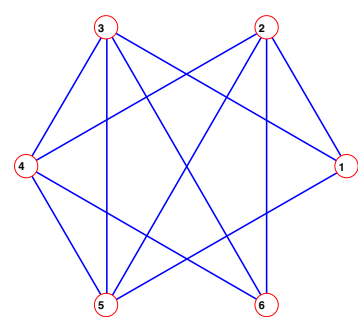

Fig. 3. The original graph is identical to its identified twin.

\section{CONCLUSION}

In this paper, we introduced a network identification scheme which involves the excitation and observation of nodes running consensus-type coordination protocols. Starting with the number of vertices in the network as a known parameter, as well as the controllability and observability of the resulting steered-and-observed network, the proposed procedure strives to collect pertinent information on the topology of the underlying graph. In this direction, we examined the applications of spectral characterization of graphs as well as a similarity transformation approach. The proposed procedure finds the original graph up to an isomorphism compatible with the input-output data.

\section{REFERENCES}

[1] O. Marques, T. Drummond, and D. Vasco, "A computational strategy for the solution of large linear inverse problems in geophysics," in Parallel and Distributed Processing Symposium, April 2003, pp. 2226.

[2] J. Mueller, S. Siltanen, and D. Isaacson, "A direct reconstruction algorithm for electrical impedance tomography," IEEE Transactions on Medical Imaging, vol. 21, no. 6, pp. 555-559, 2002.

[3] E. B. Curtis and J. A. Morrow, Inverse problems for electrical networks. USA: World Scientific, 2000.

[4] M. Kac, "Can one hear the shape of a drum?" The American Mathematical Monthly, vol. 73, no. 4, 1966.

[5] B. Gutkin and U. Smilansky, "Can one hear the shape of a graph?" Journal of Physics A: Mathematical and General, vol. 34, pp. 60616068, 2001.

[6] M. Mesbahi and M. Egerstedt, Graph Theoretic Methods for MultiAgent Networks. Princeton University Press, 2010.

[7] M. Nabi-Abdolyousefi and M. Mesbahi, "Network identification via node knock-out," in 49th IEEE Conference on Decision and Control, dec. 2010, pp. $2239-2244$.

[8] — "A sieve method for consensus-type network tomography," IET Control Theory \& Applications, To appear.

[9] A. Fujita, J. R. Sato, H. M. Garay-Malpartida, R. Yamaguchi, S. Miyano, M. C. Sogayar, and C. E. Ferreira, "Modeling gene expression regulatory networks with the sparse vector autoregressive model," BMC Systems Biology, vol. 1, 2007.

[10] D. Yu, M. Righero, and L. Kocarev, "Estimating topology of networks," Physical Review Letters, vol. 97, p. 188701, 2006.

[11] D. Napoletani and T. Sauer, "Reconstructing the topology of sparsely connected dynamical networks," Physical Review E, vol. 77, p. 26103, 2008.

[12] C. D. Michener and R. R. Sokal, "A quantitative approach to a problem in classification," Evolution, vol. 11, no. 2, pp. 130-162, 1957.

[13] Y. Yuan and J. Goncalves, "Minimal-time network reconstruction for DTLTI systems," in 49th IEEE Conference on Decision and Control, 2010, pp. 2676-2681.

[14] A. D. W. E. Moustafa, G. M. Namata and L. Getoor, "Declarative analysis of noisy information networks," in ICDE Workshop on Graph Data Management: Techniques and Applications, 2011.

[15] S. K. G. M. Namata and L. Getoor, "Collective graph identification," in ACM SIGKDD International Conference on Knowledge Discovery and Data Mining, 2011.

[16] E. T. E. Zheleva and L. Getoor, Privacy in Social Networks, Synthesis Lectures on Data Mining Series. Morgan and Claypool Publishers, 2012.

[17] D. Materassi and G. Innocenti, "Topological identification in networks of dynamical systems," IEEE Transactions on Automatic Control, vol. 55 , no. 8 , pp. $1860-1871,2010$

[18] _ _ "Unveiling the connectivity structure of financial networks via high-frequency analysis," Physica A: Statistical Mechanics and its Applications, vol. 388, no. 18, pp. 3866-3878, 2009.

[19] M. Ayazoglu, M. Sznaier, and N. Ozay, "Blind identification of sparse dynamic networks and applications," in 50th IEEE Conference on Decision and Control, Dec. 2011, pp. 2944-2950.

[20] B. M. Sanandaji, T. L. Vincent, and M. B. Wakin, "Exact topology identification of large-scale interconnected dynamical systems from compressive observations," in American Control Conference, 2011.

[21] C. D. Godsil, "Controllablity on networks," http://quoll.uwaterloo.ca/mine/Talks/control.pdf.

[22] R. A. Horn and C. R. Johnson, Matrix Analysis. Cambridge University Press, 1985.

[23] N. J. Higham, "Computing a nearest symmetric positive semidefinite matrix," Linear Algebra and its Applications, vol. 103, no. 6, pp. 103118, 1988.

[24] - Matrix Nearness Problems and Applications. Oxford U. Press, 1989.

[25] G. W. Stewart and J. Sun, Matrix Perturbation Theory. Academic Press Inc, 1990.

[26] (2010) Ibm ilog cplex optimization studio. [Online]. Available: http://www-01.ibm.com/software/integration/optimization/cplexoptimization-studio/ 